\title{
Changes in chromosome organization during PHA-activation of resting human lymphocytes measured by cryo-FISH
}

\author{
Miguel R. Branco ${ }^{1}$, Tiago Branco ${ }^{2}$, Francisco Ramirez ${ }^{3} \&$ Ana Pombo ${ }^{1 *}$ \\ ${ }^{1}$ MRC Clinical Sciences Centre, Faculty of Medicine, Imperial College London, Hammersmith Hospital Campus, \\ Du Cane Road, London, W12 ONN, UK; E-mail: ana.pombo@csc.mrc.ac.uk; ${ }^{2}$ Wolfson Institute for Biomedical \\ Research, UCL, Gower Street, London, WC1E 6BT, UK; ${ }^{3}$ South Ruislip, Middlesex, UK \\ * Correspondence
}

Key words: chromosome territory, cryosections, lymphocyte activation, radial position, stereology

\begin{abstract}
During interphase, chromosomes are arranged into territories within a highly organized nuclear space containing several compartments. It is becoming clear that this complex nuclear arrangement is important for gene regulation and therefore expression. The study of chromosome organization in interphase requires highresolution imaging methods that at the same time allow for flexible labelling strategies and preserve nuclear structure. Tokuyasu cryosections of cells or tissues provide a simple, high-resolution platform for performing immunolabelling and fluorescence in situ hybridization (FISH) on well-preserved samples. Here we show how FISH performed on thin cryosections (cryo-FISH) can be used for the study of chromosome organization at high resolution and in a quantitative manner. We have measured chromosome intermingling, volume and radial position, in resting and activated human lymphocytes, and observed chromosome-specific differences between the two cellular states. These differences are in part related to the nuclear expansion that occurs during activation, but are also likely to be tied to their different transcriptional profiles. Extrapolation of our dataset to the whole genome suggests that activated cells contain a lower amount of chromatin involved in intermingling than resting cells.
\end{abstract}

\section{Introduction}

The organization of the cell nucleus has attracted much of the attention of cell and molecular biologists in recent years. It is now clear that the nucleus is a highly complex organelle, containing various nonmembranous compartments, or nuclear bodies, which constitute structural centres for different nuclear functions (Spector 2003, Handwerger \& Gall 2006).
Transcription of ribosomal genes, for example, is carried out within the nucleolus in large RNA polymerase (RNAP) I aggregates, and transcription by RNAP II and III is similarly organized, only in smaller foci (Martin \& Pombo 2003). Splicing speckles, Cajal bodies and PML bodies are some of other nuclear bodies that have roles in nuclear function, and seem to establish interactions with specific genomic loci, which may be necessary for

\section{Electronic supplementary material}

The online version of this article (doi:10.1007/s10577-008-1230-x) contains supplementary material, which is available to authorized users. 
their function (Lamond and Spector 2003, Dellaire \& Bazett-Jones 2004, Handwerger \& Gall 2006). Chromosomes are also organized into domains, termed chromosome territories (CTs), which have non-random radial positions in the nucleus that are evolutionarily conserved (Tanabe et al. 2002, Cremer et al. 2003, 2006). Nevertheless, chromosome position is tissue-specific to a certain degree, and proximity or extent of intermingling between CTs correlates with the respective potential for translocations (Parada et al. 2004, Branco \& Pombo 2006, Soutoglou et al. 2007). Chromosome organization during interphase also has important implications in gene expression. Different models of chromosome organization have tried to explain how transcription is organized within a CT, and how it relates with the various levels of chromatin folding (Dehghani et al. 2005, Cremer et al. 2006, Branco \& Pombo 2007). Namely, chromosome conformation seems to be important for the establishment of long-range functional interactions between loci up to several Mbp away, or even across different chromosomes (Tolhuis et al. 2002, Osborne et al. 2004, Lomvardas et al. 2006). These intra- and inter-chromosomal associations are often tissue-specific, and were recently found to be surprisingly frequent (Simonis et al. 2006, Wurtele \& Chartrand 2006, Zhao et al. 2006), such that they are likely to contribute to the spatial organization of chromosomes (Pombo \& Branco 2007). However, it remains unclear which of these associations are direct functional interactions, and which may simply reflect a compartmentalization of chromatin, for example, into active and inactive compartments in the nucleus (Simonis et al. 2006).

Given the small dimensions of some of the nuclear entities, and the sub-micrometer scale of functional spatial associations, experimental systems for the study of chromosome organization need to yield high-resolution images. Additionally, spatial preservation during fixation and labelling procedures are of utmost importance to accurately assess the spatial relationships between the different nuclear landmarks. Classical two-dimensional (2D) FISH protocols on whole cells severely disrupt nuclear architecture and may lead to artefacts (Hepperger et al. 2007). Spatial preservation can be improved with three-dimensional (3D) FISH protocols, at least at the light microscopy (LM) level (Solovei et al. 2002), but this comes at the cost of minimizing treatments that can improve signal intensity. In these protocols, the stringency of the fixation step is also compromised to allow probe accessibility, which potentially alters nuclear organization (Guillot et al. 2004). In addition, imaging of whole cells using optical sections provides limited resolution (at best $500 \mathrm{~nm}$ axially on a confocal microscope), although current developments in light microscopy are expected to break this limit (Hell 2007).

While the use of strong fixatives, such as those used for conventional electron microscopy (EM) studies, can provide good ultrastructural preservation (Figure 1B), it precludes probe accessibility in whole cells. Thin sectioning of cells provides accessibility, and can thus compensate for the stringent fixation step (Branco et al. 2005). Whereas most resins used for cell embedding before sectioning hinder further labelling procedures, sections of frozen sucroseembedded cells (Tokuyasu cryosections; Figure 1A) provide the same labelling flexibility as whole cells (Tokuyasu 1973, Pombo 2007). Sectioning also improves resolution in the light microscope, as the axial resolution becomes the thickness of the section (i.e. 100-200 nm; Figure 1C). Placing cryosections directly on metal grids, instead of glass coverslips, allows for their use in EM imaging without the need for additional laborious resin-embedding steps and further sectioning. In fact, LM imaging can be performed on sections mounted on metal grids, which makes them extremely useful for correlative studies, where regions of interest can first be identified by LM, at high throughput, before imaging by EM, at high resolution (Pombo et al. 1999a, Robinson et al. 2001, Branco \& Pombo 2006). Strategies for correlative microscopy using whole cells and resin embedding normally avoid the need for post-LM labelling by using, for example, fluorochromes (namely, GFP) that photooxidize 3:3'diaminoobenzidine to produce an electron-dense precipitate (Grabenbauer et al. 2005). Quantum dots, which are fluorescent and electron-dense semiconductor nanocrystals, have also been used (Nisman et al. 2004).

Highly sensitive immunolabelling protocols for cryosections have been developed and used extensively for the study of transcription factories (Pombo et al. 1999b, Branco et al. 2005, Faro-Trindade \& Cook 2006). We have recently developed cryo-FISH, a FISH protocol for cryosections that provides highresolution imaging of chromosomes and single genomic loci at the LM level and good spatial 


\section{A}

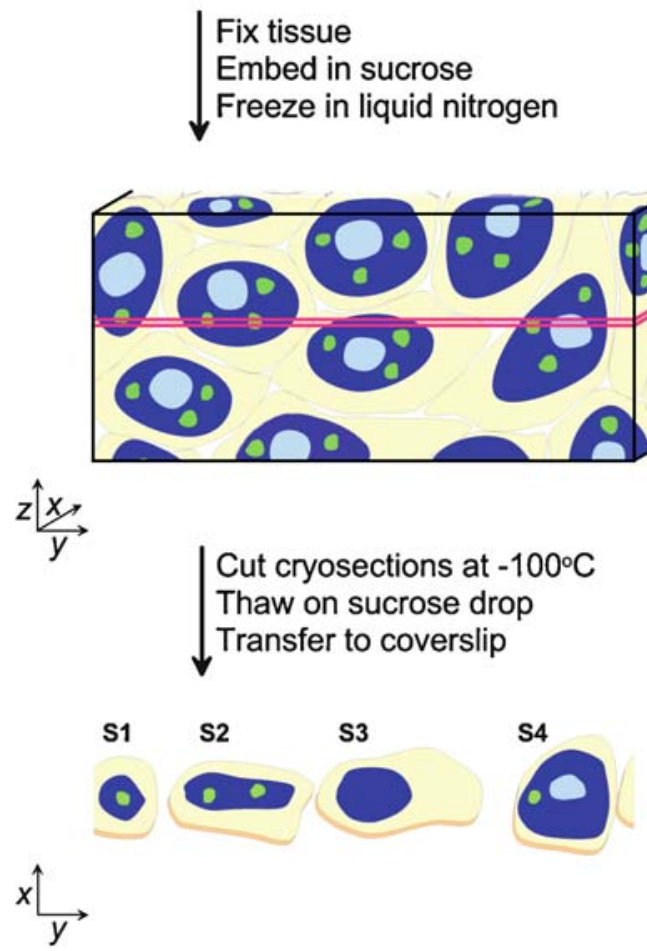

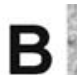
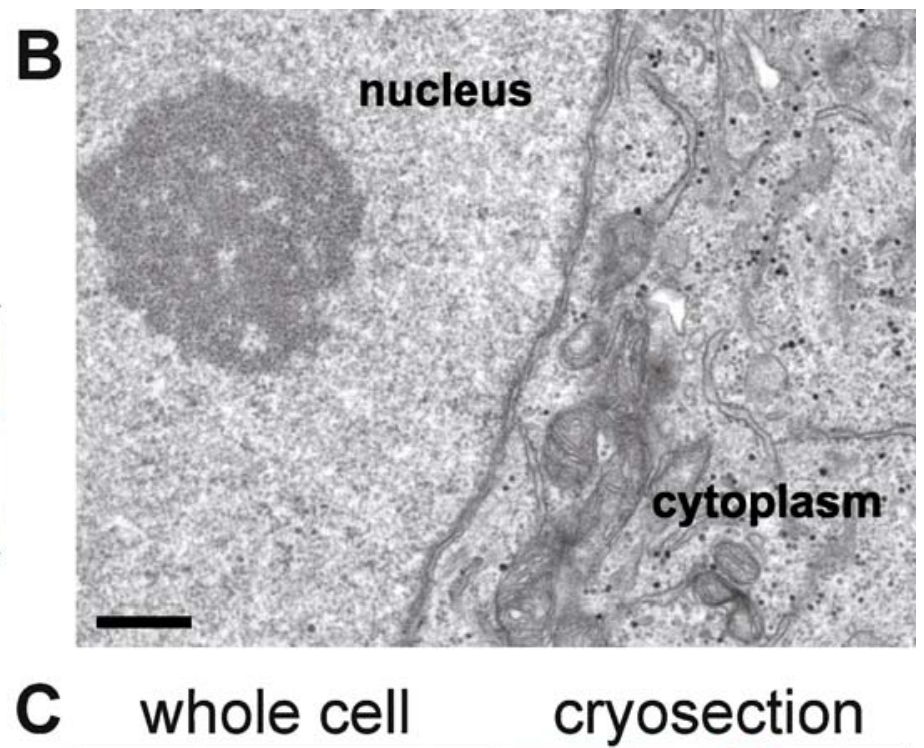
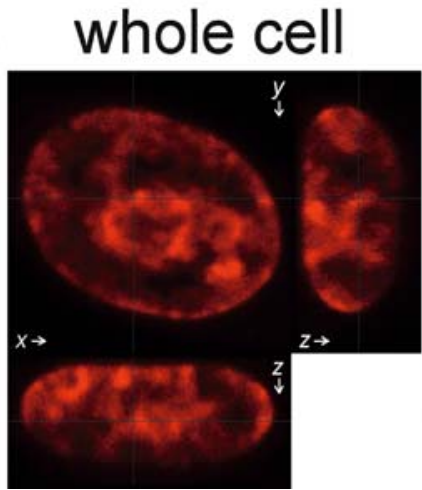

cryosection

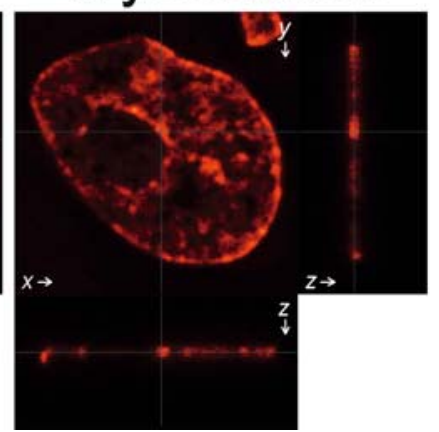

Figure 1. Tokuyasu cryosections provide optimal nuclear preservation and are compatible with high-resolution fluorescence imaging. (A) Tokuyasu cryosectioning involves chemical fixation, sucrose embedding and freezing of cells or tissues, before thin sections are cut by ultracryomicrotomy. Frozen sections are collected on sucrose drops and transferred to a solid support, and the sucrose is washed away before labelling with antibodies or nucleic acid probes. The different cellular profiles present in a cryosection (along red lines; S1-4) originate from different cells in the tissue and represent different parts of the nucleus. Pale yellow, cytoplasm; dark blue, nucleus; light blue, nucleoli; green, splicing speckles. (B) Cryosections are obtained from cells fixed in conditions that preserve cellular ultrastructure. HeLa cells were fixed as for cryosectioning, stained and Epon-embedded (Guillot et al. 2004); imaging by electron microscopy shows good preservation of membrane integrity, organelles (e.g. mitochondria) and nucleolus. Bar $=500 \mathrm{~nm}$. (C) Thin cryosections provide optimal imaging conditions for high resolution and contrast on fluorescence microscopy. 3D stacks were collected from whole HeLa cells or cryosections stained with TOTO-3, a nucleic acid dye. (Adapted from Pombo (2007) with permission.)

preservation of chromatin at the nanometer scale, and that can be used for correlative EM imaging (Branco $\&$ Pombo 2006). The use of a stronger fixative than in conventional 3D-FISH protocols increases the resistance of cryosections to the harsh treatments used to improve probe accessibility and therefore signal intensity and coverage, while retaining spatial preservation of the chromatin (Branco \& Pombo 2006). One disadvantage of cryo-FISH is that $3 \mathrm{D}$ information is lost for structures larger than the thickness of the section. As serial sectioning and reconstruction of whole cells is technically very challenging, each cell is typically represented by a single random section, which can make difficult the quantification of large-scale parameters, such as radial position. However, the mathematical tools of stereology, which were initially developed to deal with quantification problems on electron-micrographs (Weibel 1979), can be readily applied to cryo-FISH images.

We have previously used cryo-FISH to study chromosome organization in phytohaemagglutinin (PHA)-activated human lymphocytes, and showed that CTs intermingle significantly, mostly at their 
edges (Branco \& Pombo 2006). The amount of intermingling across different pairs of CTs correlated with their translocation potential and changed in a CT-specific fashion upon inhibition of transcription. This suggested that transcription may mediate interchromosomal associations and that these are significant enough to influence chromosome organization at least at the local level (Branco \& Pombo 2006, 2007). Cryo-FISH has also been used to detect longrange interactions between individual genomic loci (Simonis et al. 2006; M. R. Branco and A. Pombo, unpublished) and to study changes in the position of genomic loci in relation to their CTs and to transcription factories (Branco \& Pombo 2006, Noordermer et al. 2008).

Here we investigate differences in CT organization between resting and PHA-activated lymphocytes, in particular at the level of chromosome intermingling, but also chromosome volume and nuclear radial position. Stimulation of resting human lymphocytes with PHA increases nuclear size and causes largescale decondensation of chromatin, with concomitant transcriptional activation (Kysela et al. 2005), leading to distinct transcriptional profiles between resting and activated lymphocytes (Torelli et al. 1981). These changes that occur during lymphocyte activation are likely to depend on and influence the spatial organization of chromosomes.

\section{Materials and methods}

\section{Cell culture, fixation, and cryosectioning}

Human female peripheral blood mononuclear cells were purified by a Leuco-Sep Separation Media (Human; Harlan Sera-Lab, Loughborough, UK) density gradient centrifugation $(500 \mathrm{~g}, 30 \mathrm{~min})$. Cells were either directly processed for cryosectioning or grown in RPMI-1640 medium containing 5\% heatinactivated FCS, $5 \mathrm{mM}$ sodium pyruvate, $2 \mathrm{mM}$ glutamine, $50 \mathrm{IU} / \mathrm{ml}$ penicillin, $50 \mu \mathrm{g} / \mathrm{ml}$ streptomycin (all from Life Technologies, Paisley, UK), $50 \mu \mathrm{g} / \mathrm{ml}$ $\beta$-mercaptoethanol (Sigma, Dorset, UK), and $5 \mu \mathrm{g} / \mathrm{ml}$ PHA (Sigma) for $72 \mathrm{~h}$. For the preparation of cell blocks for cryosectioning, cells were fixed in $4 \%$ and then $8 \%$ paraformaldehyde in $250 \mathrm{mM}$ Hepes $\mathrm{pH} 7.6$ (10 min and $2 \mathrm{~h}$, respectively; Guillot et al. 2004). Cell pellets were embedded in $2.1 \mathrm{M}$ sucrose in phosphate-buffered saline (PBS) and frozen in liquid nitrogen as described previously (Pombo et al. 1999b). Cryosections were cut using an UltraCut UCT 52 ultracryomicrotome (Leica, Milton Keynes, UK), captured in sucrose drops, and transferred to coverslips. Sections on sucrose drops were stored at $-20^{\circ} \mathrm{C}$.

\section{Immunolabelling}

For immunolabelling, cryosections were washed in PBS, incubated in $0.1 \%$ Triton $\mathrm{X}-100$ in PBS (10 min), and labelled as described previously (Pombo et al. 1999b), except that PBS+ contained $0.1 \%$ casein. Serine 2 phosphorylated RNAP II was indirectly immunolabelled with H5 (1/1000; Covance, Berkeley, CA, USA).

\section{Cryo-FISH}

Directly labelled (Rhodamine, Texas Red, or FITC) whole human chromosome paints (Qbiogene, Cambridge, UK) were corrected for low-level background by addition of human Cot1 DNA (Roche; 1.7 or $3.3 \mu \mathrm{g} / \mu \mathrm{l}$ final concentration), denatured at $70^{\circ} \mathrm{C}$ for $10 \mathrm{~min}$, and re-annealed at $37^{\circ} \mathrm{C}$ for $30 \mathrm{~min}$ before hybridization. After washing with PBS, cryosections were incubated at $37^{\circ} \mathrm{C}$ with $250 \mu \mathrm{g} / \mathrm{ml}$ RNase A (1 h), treated with $0.1 \mathrm{M} \mathrm{HCl}(10 \mathrm{~min})$, dehydrated in ethanol (30-100\% series, on ice, $3 \mathrm{~min}$ each), heat-denatured $\left(8 \mathrm{~min}, 80^{\circ} \mathrm{C}\right)$ in $70 \%$ deionized formamide in $2 \times \mathrm{SSC}$, and dehydrated as above, before probe was added. Hybridization was carried out at $37^{\circ} \mathrm{C}$ for $>40 \mathrm{~h}$. Post-hybridization washes were as follows: $50 \%$ formamide in $2 \times \mathrm{SSC} 42^{\circ} \mathrm{C}$ $(3 \times$ over $25 \mathrm{~min}), 0.1 \times \mathrm{SSC}\left(60^{\circ} \mathrm{C}, 3 \times\right.$ over $30 \mathrm{~min})$, and $4 \times \mathrm{SSC}$ with $0.1 \%$ Tween $-20\left(42^{\circ} \mathrm{C}\right.$, $10 \mathrm{~min})$. Nuclei were counterstained with $2 \mu \mathrm{M}$ TOTO-3 or $20 \mathrm{ng} / \mathrm{ml}$ DAPI in PBS/0.1\% Tween-20 and coverslips were mounted in Vectashield (Vector Laboratories, Peterborough, UK).

\section{Microscopy}

For confocal laser scanning microscopy, images were collected sequentially on a Leica TCS SP2 (100X PL APO 1.40 oil objective) equipped with argon $(488 \mathrm{~nm})$ and HeNe (543 nm; $633 \mathrm{~nm})$ lasers, or a Leica TCS SP1 (100X PL APO 1.35 oil objective) equipped with UV (351/364 nm), argon (488 nm), 
krypton $(568 \mathrm{~nm})$ and $\mathrm{HeNe}(633 \mathrm{~nm})$ lasers. For wide-field LM, images were collected sequentially on a Delta-Vision Spectris system (Applied Precision, Issaquah, WA, USA) equipped with an Olympus IX70 wide-field microscope (100X UPlanFl 1.3 oil objective), a CCD camera, and the following filters: DAPI, FITC, RD-TR-PE, CY-5, CFP, YFP. No bleedthrough was detected in these conditions. The use of ultrathin cryosections allows for the use of wide-field microscopy with no reduction in axial $(z)$ resolution and only a small reduction in lateral resolution (Pombo et al. 1999a).

\section{Image analysis and measurements}

Images were manually thresholded in Adobe Photoshop (Adobe Systems, Edinburgh, UK) to define masks for nuclei or CTs. Threshold values were chosen empirically so that the entire CT was selected but no widespread nuclear background was included (see Supplementary Figure S1). Independent threshold selection by four different people has shown that this variability is in the same order of magnitude as the variability obtained across independent experiments (Branco \& Pombo 2006).

CT and intermingling volumes were calculated according to stereological methods (Weibel 1979) after collecting random images of nuclear sections. CT or intermingling areas were averaged across all sections and divided by the average of the nuclear areas. This ratio $(R)$ is equivalent to the ratio of the respective average volumes, as shown here:

$\frac{A_{\mathrm{ROI}}}{A_{\mathrm{NUC}}}=\frac{t \times A_{\mathrm{ROI}}}{t \times A_{\mathrm{NUC}}}=\frac{V_{\mathrm{ROI}}}{V_{\mathrm{NUC}}}=R$

where $A_{\mathrm{ROI}}$ is the average $\mathrm{CT}$ or intermingling area, $A_{\mathrm{NUC}}$ is the average nuclear area, $V_{\mathrm{ROI}}$ and $V_{\mathrm{NUC}}$ are the corresponding average volumes, and $t$ the section thickness. Using average section volumes for $R$ gives the same result as using average whole-nuclei volumes if enough random sections from different cells are included in the calculation. To obtain several values for $R$ within one hybridization experiment (to allow statistical analysis), images were randomly grouped and $R$ was calculated for each group. Standard deviations remained constant with increasing number of groups until a group size was reached at which $R$ did not contain enough informa- tion and the standard deviation increased abruptly. The highest number of groups before this increase was used. Group size varied between different chromosome pairs, averaging 55 sections per group, and up to 4 groups were used in an experiment (a total of 57 to 211 sections were analysed in individual experiments). Standard deviations obtained by this method were consistent with standard deviations between independent hybridization experiments.

For the measurement of CT radial position, we developed an algorithm in MatLab (The MathWorks, Inc., Natick, MA, USA) that first uses the thresholdbased masks of each nuclear section to calculate their radii. After estimating the axial position of each section based on the 3D average nuclear radius, it predicts the area that each of ten $3 \mathrm{D}$ concentric shells should occupy in that section. Masks are then generated by an interpolation algorithm so that each shell has the same shape as the section, and occupies the predicted area (Figure 5B). Fluorescence intensities of the chromosome paints are then measured for each shell and plotted against the nuclear radius (Figure 5C). For a large enough group of sections, an average radial position can be obtained from this distribution, which allows for the calculation of standard deviations across groups of sections. The same groups of sections defined for intermingling analysis were used to obtain independent measurements of the average CT radial position.

The sequential subtraction method was used to obtain a distribution of 3D nuclear radii from a population of random cryosections (Weibel 1979). Upon sectioning, each nucleus gives rise to a series of differently sized sections such that a polar section from a larger nucleus can have the same size as an equatorial section from a smaller nucleus. The sequential subtraction method aims to discard the contribution of all polar sections from the distribution of section sizes, leaving only the equatorial sections, which represent the true size of the 3D population. From the distribution of section sizes, the histogram class corresponding to the largest sections is assumed to be from equatorial sections of the largest nuclei. The expected frequency of polar sections generated by the sectioning of these nuclei is calculated and subtracted from the distribution (see Supplementary Figure S2). The next largest size category can then be assumed not to contain any polar sections, and the same steps are carried out sequentially for all size categories, after which only 

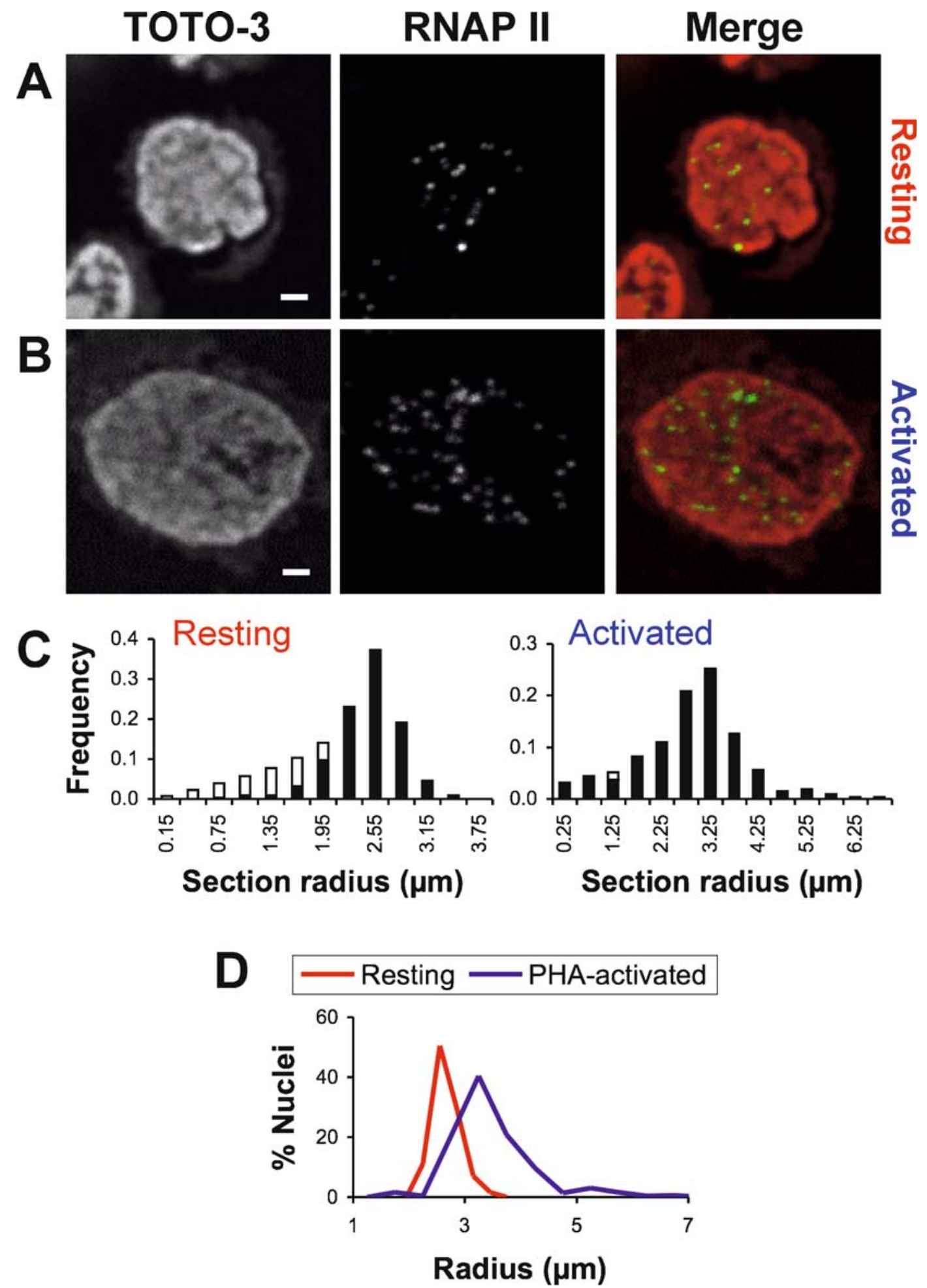
the sizes of equatorial sections will be represented in the final size distribution.

\section{Statistical analyses}

Statistical analyses were performed using factorial analysis of variance (ANOVA) in SAS software (v. 9.1). Statistically significant levels were 0.05 and 0.10 for main effects and interactions, respectively. Assumptions for the ANOVA model, i.e. constant variance and normal distribution of residuals, were confirmed by graphical diagnostic tests. Comparisons of differences in intermingling, volume and position between resting and activated samples were performed with Student's t-test using the estimates from the ANOVA models. Level of significance was 0.05 .

\section{Results}

\section{Morphological and transcriptional changes upon PHA-activation of human lymphocytes}

The first noticeable change that occurs upon activation of resting human lymphocytes by PHA is an increase in nuclear volume. To quantify this difference, we used a stereological algorithm named the sequential subtraction method (Weibel 1979), which is suitable for the analysis of sections from round nuclei. Using experimental measurements of radii from the population of random nuclear sections, the sequential subtraction method allows estimation of the distribution of 3D radii in the original cell population from which sections were cut. For each cell type, the radii of nuclear sections were measured after staining with DAPI or TOTO-3 and the corresponding distribution of radii of the $3 \mathrm{D}$ cell population was calculated (Figure 2). As expected, resting cells have smaller nuclei than activated cells, with average radii of $2.7 \pm 0.3$ and $3.5 \pm 0.8 \mu \mathrm{m}$, respectively ( $p<0.0001: n=309$ and 317 nuclear sections for resting and activated cells, respectively). This is translated into an $\sim 2$-fold increase in nuclear volume upon activation of resting lymphocytes (from 82 to $180 \mu \mathrm{m}^{3}$ ). These results are in accordance with values obtained before and after concavalin A activation (Dardick et al. 1981), but differ from recent measurements of PHA-activated cells based on the identification of equatorial sections from resin-embedded EM samples (Kysela et al. 2005). The increase in nuclear size accompanies the largescale chromatin decondensation that is associated with an increase in transcriptional activity. In fact, the number of foci containing the active form of RNAP II in activated cells is notably higher than that in resting lymphocytes (Figure 2A,B).

\section{Effect of activation on CT volume and intermingling}

States of transcriptional activity or silencing are thought to be a major determinant for the establishment of interchromatin associations, but it is unclear what the influence of such associations is on the conformation and interaction between CTs in cells with different transcriptional states. For example, the higher transcriptional activity of activated cells might yield more functional interchromatin associations in this cell population when compared with resting cells. We envisaged that $\mathrm{CT}$ intermingling might be lower in activated cells if more intrachromosomal associations that stabilize CT conformation were present, and conversely higher if more interchromosomal associations existed. Using cryo-FISH, we have previously found that CTs intermingle significantly in nuclei of activated lymphocytes (Branco \& Pombo 2006, see also Figure 3D-F). We therefore tested whether CTs in resting cells intermingle to a

Figure 2. Changes in nuclear volume and transcriptional activity upon activation of resting lymphocytes. (A, B) The serine 2 phosphorylated form of RNAP II was immunolabelled in cryosections of resting (A) and PHA-activated (B) human lymphocytes, and counterstained with TOTO-3. Upon activation of resting cells, the number of active RNAP II foci increased concomitantly with the nuclear enlargement and transcriptional activation. Bars $=1 \mu \mathrm{m}$. (C, D) To measure the 3D radius of the nucleus in the two cell populations, the radii of nuclear sections of resting and activated lymphocytes were measured after staining with DAPI or TOTO-3 ( $n \geq 309$ nuclear profiles; (C)). The sequential subtraction method (Weibel 1979) was applied to account for the contribution of polar sections in the distribution. Solid bars represent the distribution of the measured nuclear profiles; open bars represent missing polar sections, as predicted by the sequential subtraction method. The values on the $x$-axes represent the average class size (note that the $x$-axes are different between the two histograms). From the frequencies of nuclear section radii, a distribution of $3 \mathrm{D}$ radii for both cell types was obtained (D), showing a difference that is translated into an $\sim 2$-fold difference in volume between resting and activated lymphocytes. 

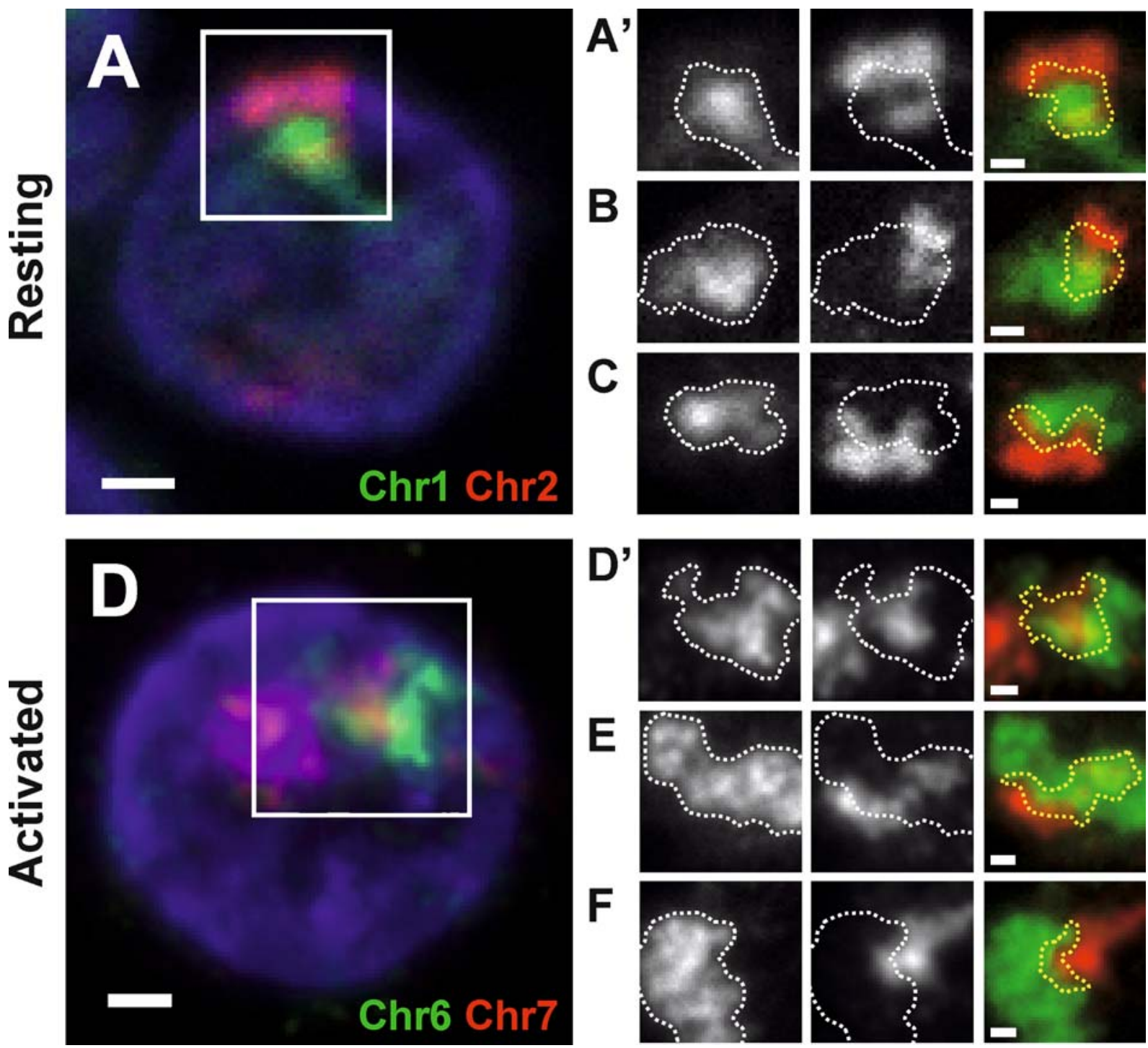

$\mathbf{F}$
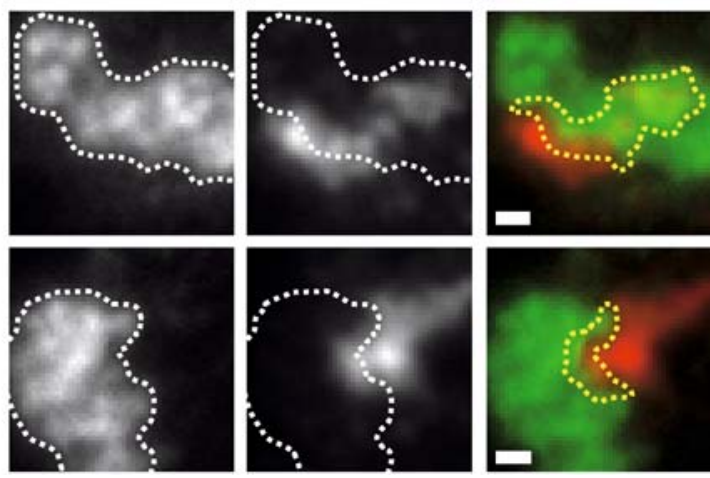

Figure 3. Chromosome intermingling in resting and activated lymphocytes. Chromosomes were painted in cryosections of resting (A-C) and PHA-activated (D-F) human lymphocytes and visualized by light microscopy. Examples of nuclear sections (blue) containing two intermingled chromosomes (green and red) are shown (A, D). Intermingling is best seen on greyscale images after the mask for one chromosome (white line) is overlaid on the image of the other chromosome $\left(\mathrm{A}^{\prime}-\mathrm{C}, \mathrm{D}^{\prime}-\mathrm{F}\right)$. The intersection between masks for both chromosomes is shown on the merged images (yellow line), representing areas of intermingling. Bars $=1 \mu \mathrm{m}(\mathrm{A}, \mathrm{D})$ and $0.5 \mu \mathrm{m}\left(\mathrm{A}^{\prime}-\mathrm{C}\right.$, $\left.\mathrm{D}^{\prime}-\mathrm{F}\right)$.

similar extent, or whether differences in nuclear volume and transcriptional activity could prevent or otherwise favour intermingling. We performed cryoFISH on sections of resting human lymphocytes using several pairs of whole chromosome paints and identified nuclear profiles with overlapping signals after imaging on the LM, as described previously (Figure 3A-C; Branco \& Pombo 2006). Chromosomes in resting cells appeared to have a similar propensity for intermingling as those in activated cells, with intermingling occurring mostly at the edges of CTs and to varying levels. We then quantified intermingling volumes for nine different pairs of chromosomes, using a simple stereological principle, which yields values that are directly normalized to nuclear volume (Figure 4A; Supplementary Table S1). Differences in relative intermingling volumes were observed between the two 
A

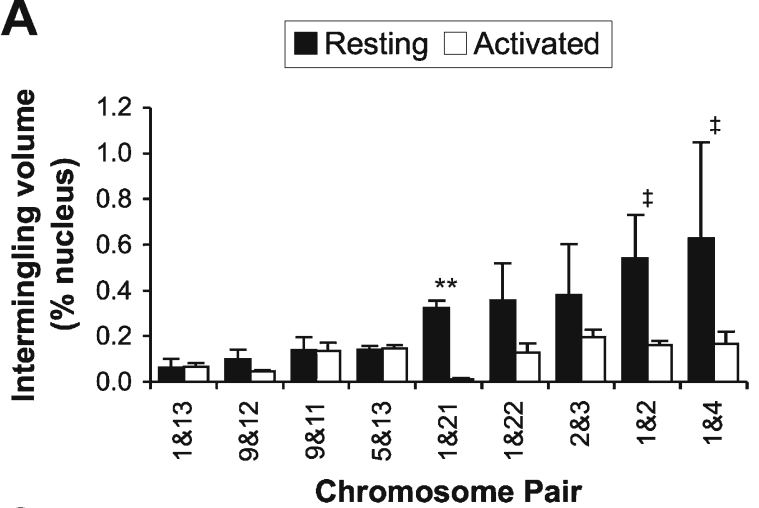

C

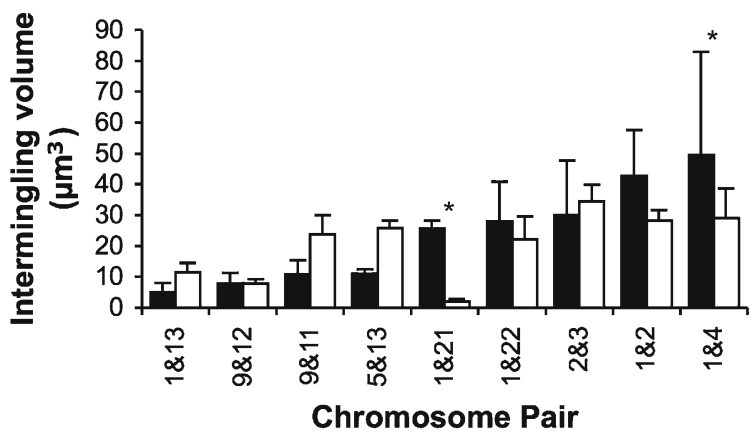

B

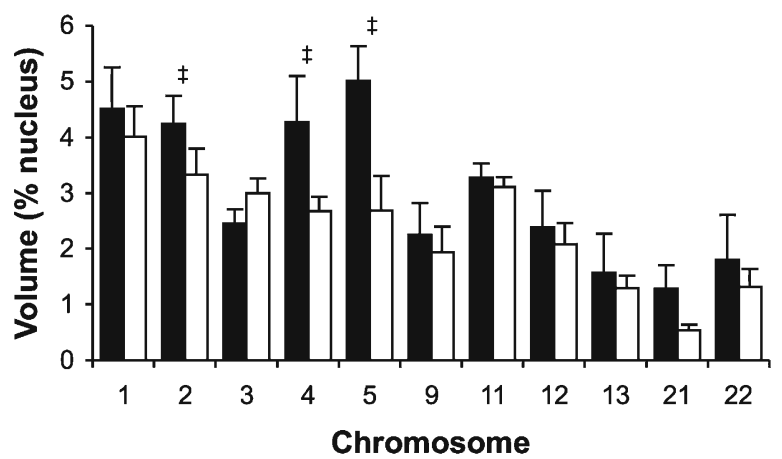

D

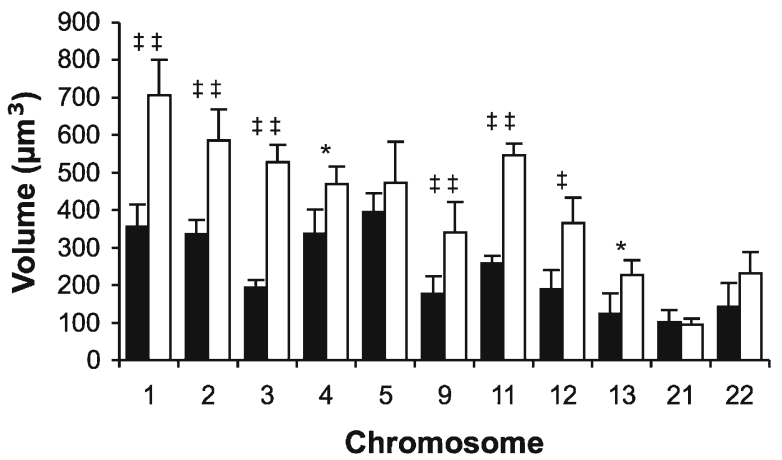

Figure 4. Differences in CT intermingling and volume between resting and activated lymphocytes. (A, B) After performing cryo-FISH on resting and activated lymphocytes, intermingling (A) and CT $(\mathbf{B})$ volumes were measured. Note that the values are normalized to the respective nuclear volumes. (C, D) To obtain absolute values of intermingling and CT volumes, the relative values were multiplied by the average nuclear volume of the corresponding cell population (see text). $* p<0.05$; ** $p<0.01 ; \ddagger p<0.001$; $\$ \ddagger p<0.0001$. Error bars represent standard deviations.

cellular states, with various pairs of chromosomes displaying a smaller extent of relative intermingling in activated cells. In fact, ANOVA testing showed that the overall effect of activation on relative intermingling was statistically significant $(p<0.0001)$, but that there were also specific changes for individual chromosome pairs $(p=0.03$ : interaction between the variables 'activation' and 'chromosome pair'). Pairwise comparisons showed that chromosome pairs $1 \& 21,1 \& 2$ and $1 \& 4$ had significantly smaller relative intermingling values in activated than in resting cells. Pairs $1 \& 22$ and $2 \& 3$ showed less significant decreases $(p=0.08$ and 0.11 , respectively). To test whether changes in relative CT volume (i.e., normalized to nuclear size) during lymphocyte activation could explain the observed differences in chromosome intermingling between the two cellular states, we measured the volumes of the CTs tested for intermingling in resting and activated cells (Figure 4B; Supplementary Table S2). PHA activation caused an overall decrease in relative CT volumes $(p<0.0001)$, with chromosomes 2,4 and 5 showing significantly smaller relative volumes in activated cells than in resting lymphocytes. Chromosome 21 also showed a large, nearly statistically significant difference $(p=0.054)$. These changes in relative CT volumes are likely to contribute to the observed differences in intermingling, e.g., the decrease in the relative volume of chromosome 2 could justify the corresponding decrease in intermingling for the pair $1 \& 2$. On the other hand, the intermingling between chromosomes 5 and 13 did not change, despite the nearly 2 -fold decrease in the relative volume of chromosome 5. This may be due to other concomitant changes that occur during activation, for example, at the level of CT position or functional associations with neighbouring CTs.

To gain further insights into the dynamic changes that occur during lymphocyte activation in terms of chromosome volumes and intermingling, we used the 
average nuclear volumes measured above for resting and activated cells (Figure 3A-C) to calculate absolute values for intermingling and chromosome volumes (Figure 4C,D; Supplementary Tables S1 and S2). As expected, most chromosome volumes are larger in activated cells than in resting cells $(p<$ 0.0001), reflecting the overall chromatin decondensation that occurs upon activation (Figure 4D). Nevertheless, this effect was chromosome-specific $(p<0.0001$ : interaction between the variables 'activation' and 'chromosome'), and three of the chromosomes analysed showed no statistically significant differences in volume (5, 21 and 22). This suggests that, although the volume of many CTs accompanies the nuclear enlargement during activation with a proportional increase in size, others do not decondense as much. Absolute intermingling volumes did not show an overall change upon activation $(p=0.4)$, although specific effects were still observed for pairs $1 \& 21$ and $1 \& 4$, where there was a statistically significant decrease in absolute intermingling volume (Figure 4C). The overall decondensation of CTs in the absence of changes in absolute intermingling suggests that a smaller fraction of chromatin from each CT intermingles in activated cells, although this would have to be confirmed by analysing a larger sample of chromosome pairs. One possibility to explain the maintenance of absolute intermingling is that the overall expansion of the CTs that occurs upon activation is not proportional to the increase in nuclear volume (as suggested by the overall decrease in relative $\mathrm{CT}$ volumes; Figure 4B), leading to a larger nuclear space being available to CTs. Interestingly, all observed effects involved changes specific to each $\mathrm{CT}$ or CT pair, suggesting a relationship with gene-
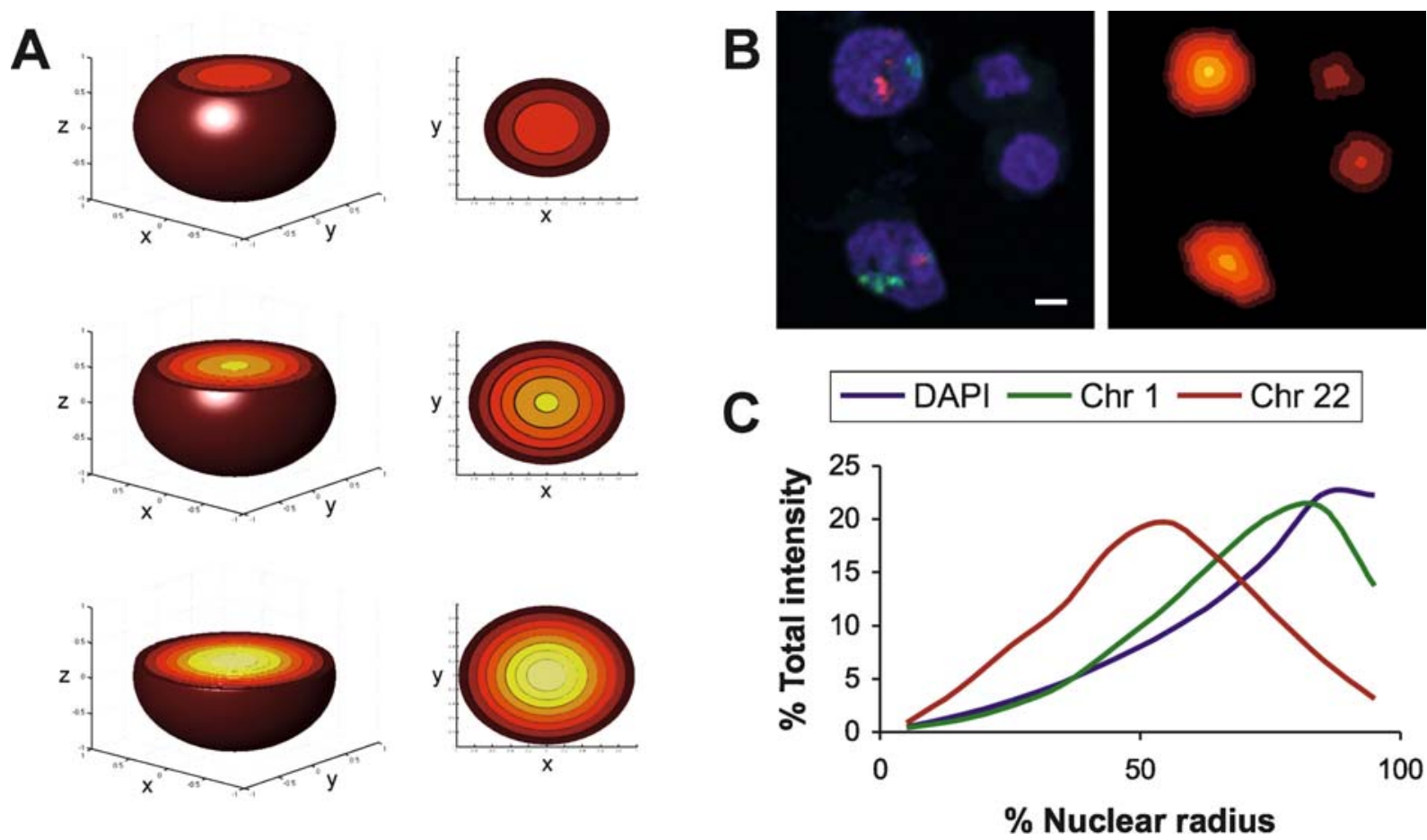

Figure 5. Measuring 3D radial position of CTs in cryosections. (A) The nucleus was divided into ten concentric shells, whose distribution in each section (right) depends on its axial position in relation to the 3D nucleus (left). (B) A MatLab algorithm was developed that estimates the axial position of each section in a cryo-FISH experiment (top; chr 1 in green and chr 22 in red) and produces masks for each shell (bottom) in accordance with the axial position. Bar $=2 \mu \mathrm{m}$. (C) The fluorescence intensity of each CT in each shell is measured and compared with the total intensity across the nucleus. The example shows chr 22 in a more central position than chr 1. 

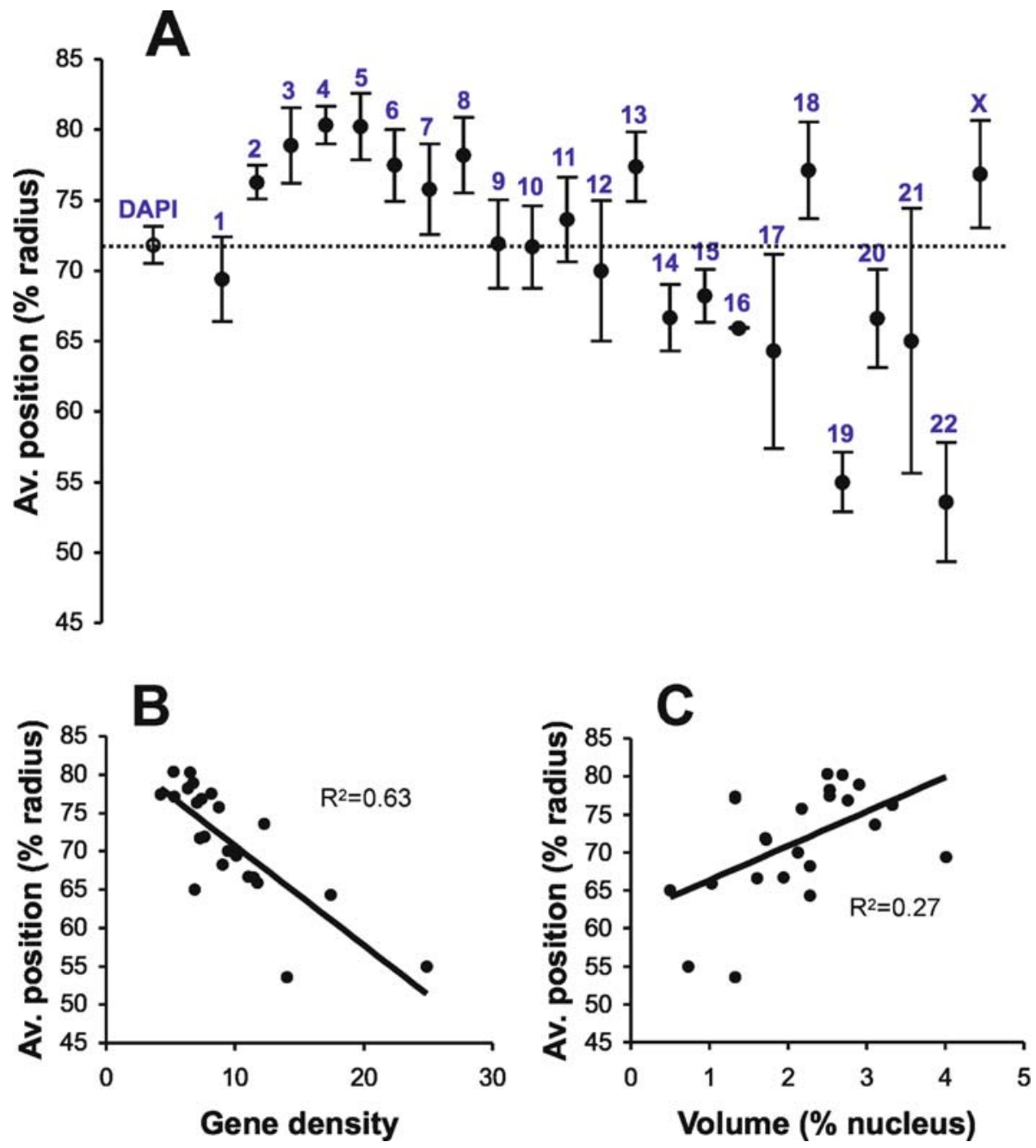

Figure 6. The radial position of all CTs in activated female lymphocytes, as seen by cryo-FISH. (A) After performing cryo-FISH on cryosections of activated lymphocytes, the average 3D radial position for all chromosomes was measured. The average radial position is represented as a percentage of the nuclear radius, such that 0 corresponds to the centre and 100 to the edge. The dotted line represents the average radial position for all chromatin, as stained by DAPI. (B) CT radial position shows a strong correlation with gene density, as seen for chromosomes labelled by 3D-FISH (Cremer et al. 2001). (C) CT radial position also has a mild correlation with chromosome volume. Error bars represent standard deviations.

specific activation of transcription during PHA stimulation.

\section{Activation-induced changes in the radial position of $C T S$}

The observation that lymphocyte activation led to chromosome-specific changes in intermingling and volume led us to investigate whether changes in radial position of CTs might create particular chromosome neighbourhoods that could influence chromosome intermingling. Chromosomal gene density and radial position are known to correlate in PHA-activated lymphocytes (Cremer et al. 2001, Croft et al. 1999), which could be driven by the profile of gene expression in each chromosome and the organization of transcription in the nucleus (Bridger et al. 2000, Parada et al. 2004). We first tested whether chromosomes labelled by cryo-FISH in activated lymphocytes exhibited the same positioning 
pattern as those labelled by 3D-FISH. For this purpose, we used a measuring principle previously developed by Cremer and colleagues, in which the nucleus is divided into concentric shells and the intensity of the respective chromosome paint is measured within each shell, thus obtaining a distribution of intensities along the nuclear radius (Cremer et al. 2001). The sections analysed were not consecutive sections of the same cells, but random sections of a large cell population, imposing a difficulty in estimating the radial position in 3D. To overcome this limitation, we first estimated the axial position $(z)$ of each section, based on its radius $(r)$ and on the average 3D nuclear radius $(R): z=\sqrt{ }\left(R^{2}-\right.$ $r^{2}$ ). Based on the estimated axial position, the distribution of the shells in each section was obtained (Figure 5A,B), and the chromosome painting intensities were measured for each shell (Figure 5C). When applied to cryo-FISH images, this method yielded a complete set of average radial positions for all chromosomes in PHA-activated female lymphocytes (Figure 6A; Supplementary Table S3). As described previously, the average radial position shows a strong correlation with gene density $\left(R^{2}=\right.$ $0.64)$, with gene-rich chromosomes preferentially located in the nuclear interior (e.g. chromosome 19), and gene-poor chromosomes at the periphery (e.g. chromosome 18; Figure 6B). Past studies have also shown a correlation with the DNA content of each chromosome, which is probably a result of geometrical constraints, given that larger chromosomes are more likely to be located in the larger nuclear periphery than at the interior (Cremer et al. 2001). To test this correlation, we plotted the average radial position against the measured $\mathrm{CT}$ volumes directly, instead of DNA content, which showed a mild correlation $\left(R^{2}=0.27\right.$; Figure 6C). Multiple regression analysis shows that both gene density and CT volume make a significant contribution to radial position ( $p<0.0001$ and $p=0.008$, respectively). To test for differences between resting and activated cells, we measured the radial positions of the 11 chromosomes analysed above in resting lymphocytes. Activation did not have an overall effect on CT radial position ( $p=0.4$ ), but specific differences were detected between the two cell populations (Figure 7; Supplementary Table S3). Although CT position also correlates with gene density in resting lymphocytes $\left(R^{2}=0.66\right.$ : versus $R^{2}=0.54$ for the same chromosomes in activated cells), two chromosomes (1 and 3) are significantly more peripherally positioned in activated cells than in resting cells (and perhaps chromosome $5, p=0.07$ ), whereas chromosome 21 becomes more centrally positioned upon activation. Note that the average relative radial distribution of chromatin (DAPI) was the same in the two cellular states, showing that the method for measuring radial position from cryosections is not affected by differences in mean and variance of the nuclear size between the two populations (see Figure 2D).

The different radial CT positions observed between resting and activated cells may be a consequence of a different network of interactions of chromo-

\section{- Resting a Activated}

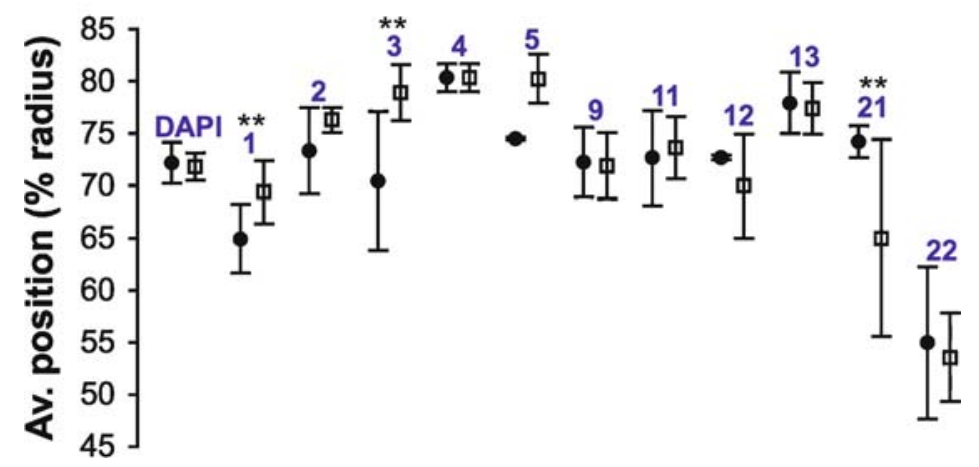

Figure 7. Differences in CT radial position between resting and activated lymphocytes. The 3D radial position of CTs tested for intermingling was measured in nuclear sections of resting lymphocytes as before, and compared with the radial distribution in activated cells. ${ }^{* *} p<0.01$. Error bars represent standard deviations. 
somes with nuclear landmarks and/or other chromosomes between the two cell types. Differences in radial position may also affect the amount of intermingling between specific pairs of CTs. However, computer simulations using the spherical chromatin domain (SCD) model and applying two different radial position models (one random and one gene density-correlated; Kreth et al. 2004) show little difference in intermingling across chromosome pairs (G. Kreth and C. Cremer, personal communication). It remains possible that with inclusion of information on different compaction levels of gene-rich and genepoor domains in the SCD model, more striking differences would be observed.

\section{Discussion}

Activation of resting human lymphocytes by PHA leads to reprogramming of the transcriptional repertoire (Torelli et al. 1981). This generalized activation has dramatic consequences on nuclear organization, including chromatin decondensation and concomitant nuclear enlargement (Kysela et al. 2005). We found that lymphocyte activation also results in specific changes in the organization of individual CTs, at the level of intermingling, CT volume and radial position, which may reflect an adaptation to the new transcriptional program. It is possible that at least some of the differences observed upon PHA activation (namely at the level of intermingling) involve changes in intra- and interchromosomal associations, similar to what seems to occur upon transcription inhibition of activated lymphocytes (Branco \& Pombo 2006). However, the nuclear enlargement, overall chromatin decondensation, and changes in the volume and radial position of individual CTs are also likely to contribute to changes in intermingling.

The total amount of intermingling in resting cells seems to involve a larger amount of chromatin than that in activated cells, given that the relative intermingling (i.e., normalized to nuclear volume) was generally higher. An expansion of the present study involving a larger number of chromosome pairs would be necessary to confirm this hypothesis. Nevertheless, it is tempting to attribute a functional significance to this observation, since a decrease in intermingling can be seen as a protective mechanism to prevent chromosome translocations. Interestingly,
DNA damage increases during $T$ lymphocyte activation as part of a mechanism for controlled cell death (Gabdoulkhakova et al. 2007). The decrease in CT intermingling could therefore reduce the risk of translocation formation in a high DNA damage environment that could otherwise lead to cancer.

We have shown here how cryo-FISH can be used as a high-resolution approach for the study of chromosome organization on spatially well-preserved cells. We also show that limitations in quantification using random sections can be overcome by the application of simple stereological methods. An important strength of cryo-FISH that was not demonstrated here is its easy adaptability to EM studies, which can be done correlatively with LM (Branco \& Pombo 2006). The reduction in the time requirement and technical difficulties that hinder the preparation of conventional EM samples opens the doors for the study of different aspects of nuclear organization (e.g. spatial associations between genomic loci and nuclear landmarks) at the nanometer level.

\section{Acknowledgements}

We thank Rainer Sachs, Gregor Kreth and Christoph Cremer for help, and the Medical Research Council (UK) and Fundação para a Ciência e a Tecnologia (Portugal) for funding.

\section{References}

Branco MR, Pombo A (2006) Intermingling of chromosome territories in interphase suggests role in translocations and transcription-dependent associations. PLoS Biol 4: e138.

Branco MR, Pombo A (2007) Chromosome organization: new facts new models. Trends Cell Biol 17: 127-134.

Branco MR, Xie SQ, Martin S, Pombo A (2005) Correlative microscopy using Tokuyasu cryosections: applications for immunolabeling and in situ hybridization. In: D. Stephens, ed. Cell Imaging. Bristol UK, Scion.

Bridger JM, Boyle S, Kill IR, Bickmore WA (2000) Re-modelling of nuclear architecture in quiescent and senescent human fibroblasts. Curr Biol 10: 149-152.

Cremer M, von Hase J, Volm T et al. (2001) Non-random radial higher-order chromatin arrangements in nuclei of diploid human cells. Chromosome Res 9: 541-567.

Cremer M, Kupper K, Wagler B et al. (2003) Inheritance of gene density-related higher order chromatin arrangements in normal and tumor cell nuclei. J Cell Biol 162: 809-820. 
Cremer T, Cremer M, Dietzel S, Muller S, Solovei I, Fakan S (2006) Chromosome territories - a functional nuclear landscape. Curr Opin Cell Biol 18: 307-316.

Croft JA, Bridger JM, Boyle S, Perry P, Teague P, Bickmore WA (1999) Differences in the localization and morphology of chromosomes in the human nucleus. J Cell Biol 145: 1119-1131.

Dardick I, Setterfield G, Hall R, Bladon T, Little J, Kaplan G (1981) Nuclear alterations during lymphocyte transformation: relationship to the heterogeneous morphologic presentations of non-Hodgkin's lymphomas. Am J Pathol 103: 10-20.

Dehghani H, Dellaire G, Bazett-Jones DP (2005) Organization of chromatin in the interphase mammalian cell. Micron 36: 95-108.

Dellaire G, Bazett-Jones DP (2004) PML nuclear bodies: dynamic sensors of DNA damage and cellular stress. Bioessays 26: 963-977.

Faro-Trindade I, Cook PR (2006) A conserved organization of transcription during embryonic stem cell differentiation and in cells with high C value. Mol Biol Cell 17: 2910-2920.

Gabdoulkhakova A, Henriksson G, Avkhacheva N, Sofin A, Bredberg A (2007) High rate of mutation reporter gene inactivation during human T cell proliferation. Immunogenetics 59: 135-143.

Grabenbauer M, Geerts WJ, Fernadez-Rodriguez J, Hoenger A, Koster AJ, Nilsson T (2005) Correlative microscopy and electron tomography of GFP through photooxidation. Nat Methods 2: 857-862.

Guillot PV, Xie SQ, Hollinshead M, Pombo A (2004) Fixationinduced redistribution of hyperphosphorylated RNA polymerase II in the nucleus of human cells. Exp Cell Res 295: 460-468.

Handwerger KE, Gall JG (2006) Subnuclear organelles: new insights into form and function. Trends Cell Biol 16: 19-26.

Hell SW (2007) Far-field optical nanoscopy. Science 316: 1153-1158.

Hepperger C, Otten S, von Hase J, Dietzel S (2007) Preservation of large-scale chromatin structure in FISH experiments. Chromosoma 116: 117-133.

Kreth G, Finsterle J, von Hase J, Cremer M, Cremer C (2004) Radial arrangement of chromosome territories in human cell nuclei: a computer model approach based on gene density indicates a probabilistic global positioning code. Biophys $J$ 86: 2803-2812.

Kysela K, Philimonenko AA, Philimonenko VV, Janacek J, Kahle M, Hozak P (2005) Nuclear distribution of actin and myosin I depends on transcriptional activity of the cell. Histochem Cell Biol 124: 347-358.

Lamond AI, Spector DL (2003) Nuclear speckles: a model for nuclear organelles. Nat Rev Mol Cell Biol 4: 605-612.

Lomvardas S, Barnea G, Pisapia DJ, Mendelsohn M, Kirkland J, Axel R (2006) Interchromosomal interactions and olfactory receptor choice. Cell 126: 403-413.

Martin S, Pombo A (2003) Transcription factories: quantitative studies of nanostructures in the mammalian nucleus. Chromosome Res 11: 461-470.

Nisman R, Dellaire G, Ren Y, Li R, Bazett-Jones DP (2004) Application of quantum dots as probes for correlative fluorescence conventional and energy-filtered transmission electron microscopy. J Histochem Cytochem 52: 13-18.

Noordermer D, Branco MR, Splinter E et al. (2008) Transcription and chromatin organization of a housekeeping gene cluster containing an integrated $\beta$-globin locus control region. PLoS Genetics 4(3): e1000016.

Osborne CS, Chakalova L, Brown KE et al. (2004) Active genes dynamically colocalize to shared sites of ongoing transcription. Nat Genet 36: 1065-1071.

Parada LA, McQueen PG, Misteli T (2004) Tissue-specific spatial organization of genomes. Genome Biol 5: R44.

Pombo A (2007) Advances in imaging the interphase nucleus using thin cryosections. Histochem Cell Biol 128: 97-104.

Pombo A, Branco MR (2007) Functional organisation of the genome during interphase. Curr Opin Genet Dev 17: 451-455.

Pombo A, Hollinshead M, Cook PR (1999a) Bridging the resolution gap: Imaging the same transcription factories in cryosections by light and electron microscopy. J Histochem Cytochem 47: 471-480.

Pombo A, Jackson DA, Hollinshead M, Wang Z, Roeder RG, Cook PR (1999b) Regional specialization in human nuclei: visualization of discrete sites of transcription by RNA polymerase III. EMBO J 18: 2241-2253.

Robinson JM, Takizawa T, Pombo A, Cook PR (2001) Correlative fluorescence and electron microscopy on ultrathin cryosections: bridging the resolution gap. J Histochem Cytochem 49: 803-808.

Simonis M, Klous P, Splinter E et al. (2006) Nuclear organization of active and inactive chromatin domains uncovered by chromosome conformation capture-on-chip (4C). Nat Genet 38: $1348-1354$.

Solovei I, Cavallo A, Schermelleh L et al. (2002) Spatial preservation of nuclear chromatin architecture during threedimensional fluorescence in situ hybridization (3D-FISH). Exp Cell Res 276: 10-23.

Soutoglou E, Dorn JF, Sengupta K et al. (2007) Positional stability of single double-strand breaks in mammalian cells. Nat Cell Biol 9: 675-682.

Spector DL (2003) The dynamics of chromosome organization and gene regulation. Annu Rev Biochem 72: 573-608.

Tanabe H, Habermann FA, Solovei I, Cremer M, Cremer T (2002) Non-random radial arrangements of interphase chromosome territories: evolutionary considerations and functional implications. Mutat Res 504: 37-45.

Tokuyasu KT (1973) A technique for ultracryotomy of cell suspensions and tissues. $J$ Cell Biol 57: 551-565.

Tolhuis B, Palstra RJ, Splinter E, Grosveld F, de Laat W (2002) Looping and interaction between hypersensitive sites in the active beta-globin locus. Mol Cell 10: 1453-1465.

Torelli G, Ferrari S, Donelli A et al. (1981) Sequence complexity and diversity of polyadenylated RNA molecules of human normal resting PHA-stimulated and leukemic lymphocytes. Nucleic Acids Res 9: 7013-7032.

Weibel ER (1979) Stereological Methods: Practical Methods for Biological Morphometry. London: Academic Press.

Wurtele H, Chartrand P (2006) Genome-wide scanning of HoxB1associated loci in mouse ES cells using an open-ended chromosome conformation capture methodology. Chromosome Res 14: 477-495.

Zhao Z, Tavoosidana G, Sjolinder M et al. (2006) Circular chromosome conformation capture (4C) uncovers extensive networks of epigenetically regulated intra- and interchromosomal interactions. Nat Genet 38: 1341-1347. 